$\Phi=$ 筧

\title{
Generalized models of flow of a fluid with pressure-dependent viscosity through porous channels: channel entry conditions
}

\author{
M.S.Abu Zaytoon ${ }^{1}$, M.H.Hamdan ${ }^{2 *}$, Yiyun (Lisa) Xiao ${ }^{2}$ \\ ${ }^{1}$ Department of Mathematics University of Petra, Amman, Jordan \\ ${ }^{2}$ Department of Mathematics and Statistics University of New Brunswick, Saint John, N.B., Canada \\ *Corresponding author E-mail: hamdan@unb.ca
}

\begin{abstract}
The flow of fluids with pressure-dependent viscosity in free-space and in porous media is considered in this study. The interest is to employ the physical model of flow through a porous layer down an inclined plane in order to derive velocity expressions that can be used as entry conditions in the study of two-dimensional flows through free-space and through porous channels. The generalized equations of Darcy, Forchheimer and Brinkman are used in this work.
\end{abstract}

Keywords: Channel Entry Conditions; Generalized Models; Porous Media.

\section{Introduction}

The realization that viscosity of a fluid depends on pressure can be traced back to the nineteenth century and works of Stokes, [1] and Barus, [2], [3]. Dependence of viscosity on pressure has been argued to be exponential in nature, as proposed by Barus [2], [3], but many other forms, including linear and polynomial expressions, have been proposed and used in the literature (cf. [4 - 7]).

Flow of fluids with pressure-dependent viscosity has been of interest due to its applications in lubrication theory, $[8,9,10]$. The past quarter of a century, however, has witnessed an increasing interest in this type of flow through porous media. This stems from the many applications in enhanced oil recovery and carbon sequestration, [11], [12], in filtration problems, [13], and in the pharmaceutical industry [14]. These and many other applications emphasize the need to accurately model flow of fluids with pressure-dependent viscosities and to obtain solutions to initial and boundary value problems.

In seeking solutions to initial and boundary value problems in fluid dynamics, the use of one- and two-dimensional flow configurations are popular idealizations of the general three-dimensional flow problems as they provide us with valuable information and better understanding of the flow properties and the effects of fluid, flow and domain parameters. Typically, flow between parallel plates and flow in pipes and two-dimensional channels, and flow over circles provide us with the necessary benchmarks to better understand the full three-dimensional flow.

In the through two-dimensional channels and through domains with constrictions or over obstacles, for instance, velocity entry conditions are important, especially when the problem involves flow of viscous fluids. Uniform flow assumptions might no longer be valid as entry conditions to a porous channel bounded by solid walls, and the popular parabolic entry profiles of the Navier-Stokes equations are approximations at best in flow of fluids with pressure-dependent viscosities through porous channels. These situations provide us in part with motivations for this work in which we derive and document expressions for the entry conditions into two-dimensional channels when the flow is that of a pressure-dependent viscosity, and the channel is either free-space (as in the case of Navier-Stokes flow) or filled with a porous material. Entry conditions to channels when the flow is that of a fluid with constant viscosity have been discussed by Hamdan [15]. In order to achieve our objective of deriving entry profile expressions for the velocity into a two-dimensional channel, this work is organized as follows. In Section 2, we discuss equations governing the flow of a fluid with pressure-dependent viscosity both in free-space and in porous media, and make the necessary assumptions on the flow. In Section 3, we discuss the flow configutation and obtain velocity expressions. In Section 4, we consider a dozen popular viscosity-pressure relations and derive appropriate channel entry conditions. Finally, we provide concluding remarks and plans for future work.

\section{Governing equations}

The steady flow of a viscous, incompressible fluid is governed by two conservation principles, namely conservation of mass, which is expressed as an equation of velocity continuity of the form, [14]:

$\nabla \cdot \vec{v}=0$ 
And conservation of linear momentum, given by the Navier-Stokes equations, namely:

$\rho(\vec{v} \cdot \nabla) \vec{v}=-\nabla p+\nabla \cdot \mu\left(\nabla \vec{v}+(\nabla \vec{v})^{T}\right)+\rho \vec{g}$

Where $\vec{v}$ is the velocity vector field, $\rho$ is the fluid density, $\mathrm{p}$ is the pressure, $\mu$ is the fluid viscosity, $\overrightarrow{\mathrm{g}}$ is the gravitational acceleration, $\nabla$ is the gradient operator and $\nabla^{2}$ is the Laplacian. The Navier-Stokes equation are partial differential equations which describe the microscopic, local motion of viscous fluid flow in space. The term $\mu \nabla^{2} \vec{v}$ is the viscous shear term and $(\vec{v} \cdot \nabla) \vec{v}$ is the convective acceleration.

Equations (1) and (2) represent an underdetermined system of four equations in the five unknowns $\vec{v}=(\mathrm{u}, \mathrm{v}, \mathrm{w}), \mathrm{p}$ and $\mu$. In the absence of additional conservation principles to provide an additional equation, it has long been recognized that viscosity can be expressed as a function of pressure to provide an additional condition to render the governing system of equations determinate. Barus, [2], [3], provided the following relation between viscosity and pressure:

$\mu=\mu_{0} \mathrm{e}^{\alpha\left(p-p_{0}\right)}$

Where $\mu$ is fluid viscosity, $\mathrm{p}$ is pressure, $\mu_{0}$ is a reference viscosity at reference pressure $\mathrm{p}_{0}$, and $\alpha>0$ is a constant. Equations (1), (2) and (3) now represent a determinate system of five scalar equations in five unknowns.

In flow through porous media, equations governing the flow of a fluid with pressure-dependent viscosity through a porous structure are continuity equation, of the form of equation (1), and a balance of momentum equation that takes different forms depending on the porous structure, the speed of the flow and the presence or absence of macroscopic boundaries. Generalized equations of flow through porous media have been derived in [4 - 7], [12], [16 - 20] using mixture theory, thermodynamic balance, or otherwise homogenization. They are summarized in what follows.

The generalized Darcy's equation has been reported as, [20]:

$\lambda(\mathrm{p}, \overrightarrow{\mathrm{x}}) \overrightarrow{\mathrm{u}}+\nabla \mathrm{p}=\rho \overrightarrow{\mathrm{g}}$

Where

$\lambda(p, \vec{x})=\frac{\mu(p)}{k(\vec{x})}=\frac{\mu_{0}}{k(\vec{x})} \cdot \exp \left[\beta_{B}\left(p-p_{0}\right)\right]$

The function $\lambda(p, \vec{x})$ is the Darcy drag, which is a function of pressure and position, $k(\vec{x})$ is the permeability function and $\beta_{B}$ is the (experimental) Barus coefficient.

The generalized Forchheimer's equation has been reported as, [20]:

$\nabla p+\left\{\frac{\mu_{0}}{k(\vec{x})} \cdot \exp \left[\beta_{B} p\right]+\beta_{f} \rho\|\vec{u}\|\right\} \vec{u}=\rho \vec{G}$

Where

$\lambda(\overrightarrow{\mathrm{u}}, \mathrm{p}, \overrightarrow{\mathrm{x}})=\frac{\mu(\mathrm{p})}{\mathrm{k}(\overrightarrow{\mathrm{x}})}+\beta_{\mathrm{f}}\|\overrightarrow{\mathrm{u}}\|=\frac{\mu_{0}}{\mathrm{k}(\overrightarrow{\mathrm{x}})} \cdot \exp \left[\beta_{\mathrm{B}} \mathrm{p}\right]+\beta_{\mathrm{f}} \rho\|\overrightarrow{\mathrm{u}}\|$

And $\beta_{\mathrm{f}}$ is the Forchheimer drag coefficient. It is customary to write $\beta_{\mathrm{f}}=\frac{\mathrm{C}_{\mathrm{f}}}{\sqrt{\mathrm{k}(\overrightarrow{\mathrm{x}})}}$, where $\mathrm{C}_{\mathrm{f}}$ is the Ergun coefficient with value between 0.375 and 0.5 .

The Darcy and Forchheimer generalized equations are characterized with the absence of viscous shear term (laplacian), hence not compatible with the presence of solid boundaries on which a no-slip condition is imposed.

The generalized Brinkman's equation can be written in the following form, [4], [7], [16], [19]:

$\rho(\vec{v} \cdot \nabla) \vec{v}=-\nabla p+\nabla \cdot 2 \mu D(\vec{v})-\lambda(p) \vec{v}+\rho \vec{g}$

Where

$\mathrm{D}(\overrightarrow{\mathrm{v}})=\frac{1}{2}\left(\nabla \overrightarrow{\mathrm{v}}+(\nabla \overrightarrow{\mathrm{v}})^{\mathrm{T}}\right)$

Where $\lambda(p)$ denotes the pressure-dependent drag coefficient and $\rho$ is the density of the fluid. Subramanian and Rajagopal [19] rightfully state that the viscosity $\mu(p)$ is a "measure of the frictional resistance in between fluid layers" while $\lambda(p)$ is a "measure of the friction between the fluid and the solid, at the pore". Clearly, $\mu(p)$ and $\lambda(p)$ control variations in viscosity due to pressure, and variations in pressure due to variations in porous parameters.

Equation (8) does not explicitly take into account the porous medium microstructure or permeability of the medium, nor does it distinguish between one porous medium and another, or between a porous medium with constant permeability and one with a variable permeability. Kannan and Rajagopal [7] proposed and tested various forms of $\mu(p)$ and $\lambda(p)$, including exponential, linear and polynomial forms.

Alzahrani et.al. [23], considered the flow down an inclined plane through a porous medium with variable permeability, using model equations (8). It is worth noting that flow through variable permeability porous material is realistic since the pore structure is arbitrary, porosity in natural media is a variable, hence one expects permeability to depend on position in much the same way as velocity and pressure.

\section{Problem formulation and solution}

In order to derive entry conditions to a channel of with $\mathrm{h}$, we assume the channel is inclined at an angle $\vartheta$ to the horizontal, where $0 \leq \vartheta<$ $\frac{\pi}{2}$. If $\vartheta=0$, then the channel is horizontal. The flow configuration is illustrated in Fig. 1 which shows the orientation of the coordinate 
system used. It is assumed that the channel is bounded by impermeable, solid walls on which the no-slip condition is applied. Furthermore, if the channel is horizontal, $\vartheta=0$, then $\mathrm{p}=\mathrm{p}(\mathrm{x}, \mathrm{y})$ and the flow is driven by gravity and by a constant pressure gradient, $\frac{\mathrm{dp}}{\mathrm{dx}}=$ constant $<$ 0 . If $0<\vartheta<\frac{\pi}{2}$, we assume that the flow is driven by gravity and take $\frac{d p}{d x}=0$, hence $p=p(y)$. We discuss in this work flow through an inclined channel, and leave flow through a horizontal channel for future work.

For the unidirectional flow at hand, continuity equation (1) implies that $\mathrm{u}=\mathrm{u}(\mathrm{y})$. We assume that the pressure function also varies with the lateral coordinate, $k=k(y)$. Introducing the dimensionless quantities $y^{*}=\frac{y}{h} ; u^{*}=\frac{u}{U} ; k^{*}=\frac{k}{h^{2}}$, where $U$ is a characteristic velocity, then the channel is of dimensionless width of unity, the permeability function and velocity are dimensionless. Conditions on velocity, permeability and pressure, after dropping the asterisks “*”, are as follows:

$\mathrm{u}(0)=\mathrm{k}(0)=0$

$\mathrm{u}(1)=\mathrm{k}(1)=0 ; \mathrm{p}(1)=\mathrm{p}_{0}$

Where $\mathrm{p}_{0}$ is a prescribed pressure, such as atmospheric pressure.

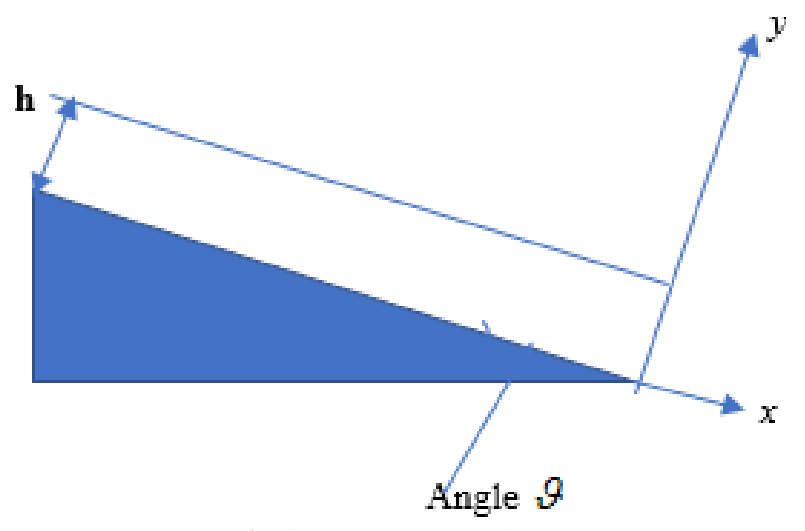

Fig. 1: Representative Sketch.

Flow in the above domain is governed by the equation of continuity (1) and one of the momentum equations (2), (4), (6) or (8), discussed in Section 2, above. If we write these equations in components' forms, then in each case the pressure can be determined from:

$-\frac{d p}{d y}-\rho g h \cos \vartheta=0$

Whose solution satisfying (11) is given by:

$p=p_{0}+(1-y) \rho g h \cos \vartheta=\left[p_{0}+\rho g h \cos \vartheta\right]-\rho g h \cos \vartheta y$

Momentum equations (2), (4), (6) and (8) are discussed in the following subsections.

\subsection{Navier-stokes flow}

Equation (2) takes the following form:

$\frac{d^{2} u}{d y^{2}}+\frac{1}{\mu} \frac{d \mu}{d y} \frac{d u}{d y}+\frac{\rho g h^{2} \sin \vartheta}{U \mu}=0$

Substituting (3) in (14), with $p-p_{0}=\rho g h \cos \vartheta(1-y)$ from (13), we can write (14) as

$\frac{d^{2} u}{d y^{2}}-A_{1} \frac{d u}{d y}+A_{3} e^{-A_{1}(1-y)}=0$

Where

$A_{1}=\alpha \rho g h \cos \vartheta$

$A_{3}=\frac{\rho g h^{2} \sin \vartheta}{U \mu_{0}}$

Solution to (15) satisfying the no-slip conditions (10) and (11) takes the form:

$u(y)=-A_{3} e^{A_{1}(y-1)}\left[\frac{y}{A_{1}}-\frac{1}{\left(A_{1}\right)^{2}}\right]+A_{3}\left[\frac{1}{\left(A_{1}\right)^{2} e^{A_{1}}}-\frac{1}{A_{1}\left(1-e^{A_{1}}\right)}\right] e^{A_{1} y}$
$+A_{3}\left[\frac{1}{\left(1-e^{A_{1}}\right)}-\frac{2}{\left(A_{1}\right)^{2} e^{A_{1}}}\right]$

This represents the entry velocity profile into a free-space channel. 


\subsection{Darcy flow}

Equations (4) and (5) yield the following generalized Darcy equation for the configuration at hand, where we have used $p-p_{0}=$ $\rho g h \cos \vartheta(1-y)$ :

$\frac{\rho g h^{2}}{U} \sin \vartheta-\left\{\frac{\mu_{0}}{k(y)} \cdot \exp \left[\beta_{B} \rho g h \cos \vartheta(1-y)\right]\right\} u=0$

Equation (19) gives the velocity profile

$u(y)=\frac{\rho g k(y) h^{2} \sin \vartheta}{U \mu_{0}} \exp \left[-\beta_{B} \rho g h \cos \vartheta(1-y)\right]$

Darcy's equation is not compatible with the presence of solid walls on which a no-slip condition is imposed. However, in equation (20), if permeability is a variable function of $y$ and is chosen such that $k(0)=k(1)=0$, then $u(0)=u(1)=0$. If permeability is constant, say $k_{0}$, then the slip velocities at the lower and upper walls are given, respectively, by

$u(0)=\frac{\rho g k_{0} h^{2} \sin \vartheta}{U \mu_{0}} \exp \left[-\beta_{B} \rho g h \cos \vartheta\right]$

And

$u(1)=\frac{\rho g k_{0} h^{2} \sin \vartheta}{U \mu_{0}}$

\subsection{Forchheimer flow}

Equations (6) and (7) yield the following generalized Forchheimer equation for the configuration at hand, where we have used $p-p_{0}=$ $\rho g h \cos \vartheta(1-y)$ :

$\rho h^{2} B_{f} U u^{2}+\left\{\frac{\mu_{0}}{k(y)} \cdot \exp \left[\beta_{B} \rho g h \cos \vartheta(1-y)\right]\right\} u=\frac{\rho g h^{2}}{U} \sin \vartheta$

Solving (23) algebraically for $u(y)$, we obtain:

$u(y)=\frac{-\left\{\frac{\mu_{0}}{k(y)} \cdot \exp \left[\beta_{B} \rho g h \cos \vartheta(1-y)\right]\right\}+\sqrt{\left\{\frac{\mu_{0}}{k(y)} \cdot \exp \left[\beta_{B} \rho g h \cos \vartheta(1-y)\right]\right\}^{2}+4 \rho^{2} h^{4} g B_{f} \sin \vartheta}}{2 \rho h^{2} B_{f} U}$

Forchheimer's equation is not compatible with the no-slip condition on solid walls. The slip velocities at the lower and upper walls are obtained from (24), respectively, as:

$u(0)=\frac{-\left\{\frac{\mu_{0}}{k(y)} \cdot \exp \left[\beta_{B} \rho g h \cos \vartheta\right]\right\}+\sqrt{\left\{\frac{\mu_{0}}{k(y)} \cdot \exp \left[\beta_{B} \rho g h \cos \vartheta\right]\right\}^{2}+4 \rho^{2} h^{4} g B_{f} \sin \vartheta}}{2 \rho h^{2} B_{f} U}$
$u(1)=\frac{-\frac{\mu_{0}}{k(y)}+\sqrt{\left\{\frac{\mu_{0}}{k(y)}\right\}^{2}+4 \rho^{2} h^{4} g B_{f} \sin \vartheta}}{2 \rho h^{2} B_{f} U}$

\subsection{Brinkman flow}

Equation (8) yields the following generalized Brinkman's equation for the configuration at hand:

$\frac{d^{2} u}{d y^{2}}+\frac{1}{\mu} \frac{d \mu}{d y} \frac{d u}{d y}+\frac{\rho g h^{2} \sin \vartheta}{U \mu}-\frac{h^{2} \lambda(p) u}{\mu}=0$

In their elegant and thorough analysis of (27), Kannan and Rajagopal [7] provided solutions using Maple for various choices of $\mu(p)$ and $\lambda(p)$ that include combinations of $A e^{\alpha p}$ and $B\left(\frac{p}{p_{0}}\right)^{n}$, where $A$ and $B$ are positive constants. Pažanin et.al. [21] employed expressions of the forms Many other forms $\lambda(p)=\lambda_{0} e^{\eta p}$, and $\mu(p)=\mu_{0} e^{\eta p}$, where $\lambda_{0}, \mu_{0}, \eta>0$.

In the current work, we assume that $\mu(p)=\mu_{0} e^{\alpha\left(p-p_{0}\right)}$ and $\lambda(p)=\lambda_{0} e^{\gamma\left(p-p_{0}\right)}$, where $\alpha, \gamma, \mu_{0}, \lambda_{0}>0$, and $p-p_{0}=\rho g h \cos \vartheta(1-y)$. Equation (27) thus takes the form:

$\frac{d^{2} u}{d y^{2}}-A_{1} \frac{d u}{d y}+A_{3} e^{-A_{1}(1-y)}-A_{2} e^{A_{4}(1-y)} u=0$

Where $A_{1}$ and $A_{3}$ are as given by (16) and (17), and

$A_{2}=\frac{h^{2} \lambda_{0}}{\mu_{0}}$

$A_{4}=(\gamma-\alpha) \rho g h \cos \vartheta$ 
Interpretations of $A_{1}, A_{2}, A_{3}$, and $A_{4}$ have been provided by Kannan and Rajagopal [7], which we directly quote so that we do not inadvertently alter the meanings, as follows:

$A_{1}$ "is a measure of the effect of gravity versus the effect of the pressure on the viscous dissipation within the fluid",

$A_{2}$ "is concerned with the relative magnitudes of the rates of dissipation within the fluid due to its viscosity versus that due to drag at the pores",

$A_{3}$ "compares the relative effects of gravity and viscosity", and

$A_{4}$ "is a measure of gravity versus the relative effects of the viscosity and the drag at the pores".

In order to solve (28) analytically, we consider the special case of $\gamma=\alpha$. This case is based on selection of viscosity and drag forms by Pažanin et.al. [21]. Equation (28) reduces to:

$\frac{d^{2} u}{d y^{2}}-A_{1} \frac{d u}{d y}-A_{2} u=A_{3} e^{-A_{1}(1-y)}=A_{3} e^{-A_{1}} e^{A_{1} y}$

Solution to (31) satisfying (9) is given by

$u=c_{1} \exp \left[\frac{A_{1}}{2}+\frac{1}{2} \sqrt{\left(A_{1}\right)^{2}+4 A_{2}}\right] y+c_{2} \exp \left[\frac{A_{1}}{2}-\frac{1}{2} \sqrt{\left(A_{1}\right)^{2}+4 A_{2}}\right] y-\frac{A_{3}}{A_{2}} e^{A_{1}(y-1)}$

Where

$$
\begin{aligned}
& c_{1}=\frac{A_{3}}{A_{2}}\left\{\frac{\exp \left(-\frac{1}{2} \sqrt{\left(A_{1}\right)^{2}+4 A_{2}}-e^{\frac{A_{1}}{2}}\right.}{e^{A_{1}}\left[\left(\exp \left(-\frac{1}{2} \sqrt{\left(A_{1}\right)^{2}+4 A_{2}}\right)-\left(\exp \left(\frac{1}{2} \sqrt{\left(A_{1}\right)^{2}+4 A_{2}}\right)\right]\right.\right.}\right\} \\
& C_{2}=\frac{A_{3}}{A_{2}}\left\{\frac{e^{\frac{A_{1}}{2}}-\left(\exp \left(\frac{1}{2} \sqrt{\left(A_{1}\right)^{2}+4 A_{2}}\right)\right.}{\left.e^{A_{1}\left[\left(\exp \left(-\frac{1}{2} \sqrt{\left(A_{1}\right)^{2}+4 A_{2}}\right)-\left(\exp \left(\frac{1}{2} \sqrt{\left(A_{1}\right)^{2}+4 A_{2}}\right)\right.\right.\right.}\right]}\right\}
\end{aligned}
$$

Taking $\gamma=\alpha$ in (28) results in $A_{4}=0$. This has the effect of increasing the velocity and bringing the velocity profile closer to that of a Brinkman velocity profile when viscosity is constant.

\section{Other forms of viscosity and drag}

There exists a large number of choices for viscosity as a function of pressure, the most popular of which are exponential, linear, and polynomial forms. Some of these are discussed in the next subsection. For the drag function, however, it is not obvious what $\lambda(p)$ should be, except possibly trying different forms. Unlike Brinkman's equation with constant viscosity and a Darcy resistance proportional to viscosity, the case of generalized Brinkman's equation possesses a drag function that seems independent of shear viscosity. It is a function of pressure, however. So is shear viscosity. In addition, for a given porous medium, it is not obvious how the drag function should be chosen so that one distinguishes between one porous medium or another, or between a porous medium with variable permeability and one with constant permeability.

To circumvent, we propose here to approximate $\lambda(p)$ by constructing a function of pressure and position that is the ratio between the pressure-dependent viscosity $\mu(p(y))$ and the permeability function $k(y)$. This idea is not strange since it appears in the velocity-independent drag term of the generalized Darcy's equation.

If we assume that $\mu=\mu(p)=f(y)$ and $\lambda(p)=\frac{\mu(p)}{k(y)}=\frac{f(y)}{k(y)}$, where $k(y)$ is the dimensionless permeability function, then equation (27) takes the form

$\frac{d^{2} u}{d y^{2}}-\rho g h \cos \vartheta \frac{f^{\prime}(p)}{f(p)} \frac{d u}{d y}-\frac{u}{k(y)}=-\frac{\rho g h^{2} \sin \vartheta}{U f(p)}$

Equation (35) is a general differential equation that governs the flow of a fluid with pressure-dependent viscosity through a porous domain down an inclined plane. Given $f(p)$ and $k(y)$, we obtain specific forms of (35).

\subsection{Forms of $\boldsymbol{f}(\boldsymbol{p})$ :}

The literature reports on many popular forms of $f(p)$, where $\mu_{0}>0, p_{0}>0, \alpha>0, b>0$ and $n$ is a positive integer. Some of these forms are:
i) $\mu(p)=f(p)=\alpha p$
ii) $\mu=f(p)=\alpha p^{n}$
iii) $\mu(p)=f(p)=\mu_{0}(1+\alpha p)$
iv) $\mu=f(p)=\mu_{0} e^{\alpha p}$
v) $\left.\mu=f(p)=\mu_{0} e^{\alpha\left(p-p_{0}\right.}\right)$
vi) $\mu=f(p)=\mu_{0}\left(1+b e^{a p}\right)$
vii) $\mu(p)=f(p)=\mu_{0}\left(1+\alpha p^{n}\right)$
viii) $\mu(p)=f(p)=\mu_{0}\left[1+\alpha\left(p-p_{0}\right)^{n}\right]$
ix) $\mu(p)=f(p)=\mu_{0}\left[1+\alpha\left(p-p_{0}\right)\right]^{n}$
x) $\mu=f(p)=\mu_{0} e^{\alpha\left(\frac{p}{p_{0}}\right)}$
xi) $\mu=f(p)=\mu_{0} e^{\alpha\left(\frac{p}{p_{0}}\right)^{n}}$
xii) $\mu=f(p)=\mu_{0}\left(p / p_{0}\right)^{n}$

Upon substituting the above forms of viscosity in (35), the following differential equations are obtained and tabulated in Table 1. 


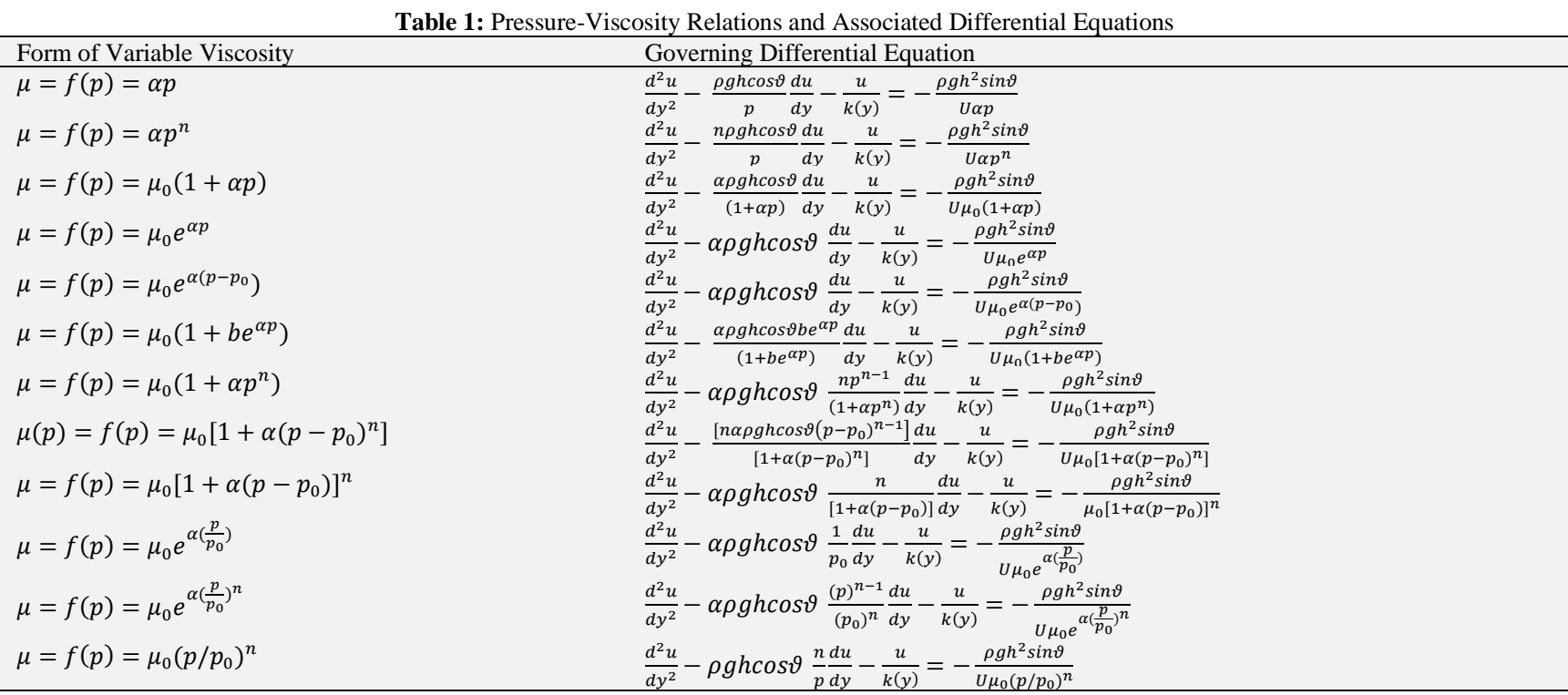

In order to cast the equations of Table 1 in more recognizable differential equations, we provide a change of variables by letting

$Y=p=p_{0}+\beta(1-y)$

$\beta=\rho g h \cos \vartheta$

Where

$\frac{d Y}{d y}=-\beta$

$\frac{d}{d y}=\frac{d}{d Y} \frac{d Y}{d y}=-\beta \frac{d}{d Y}$

$\frac{d^{2}}{d y^{2}}=\beta^{2} \frac{d^{2}}{d Y^{2}}$

The following table of transformed governing equations is obtained, wherein:

$\delta=\rho g h^{2} \sin \vartheta$

Table 2: Transformed Governing Differential Equations

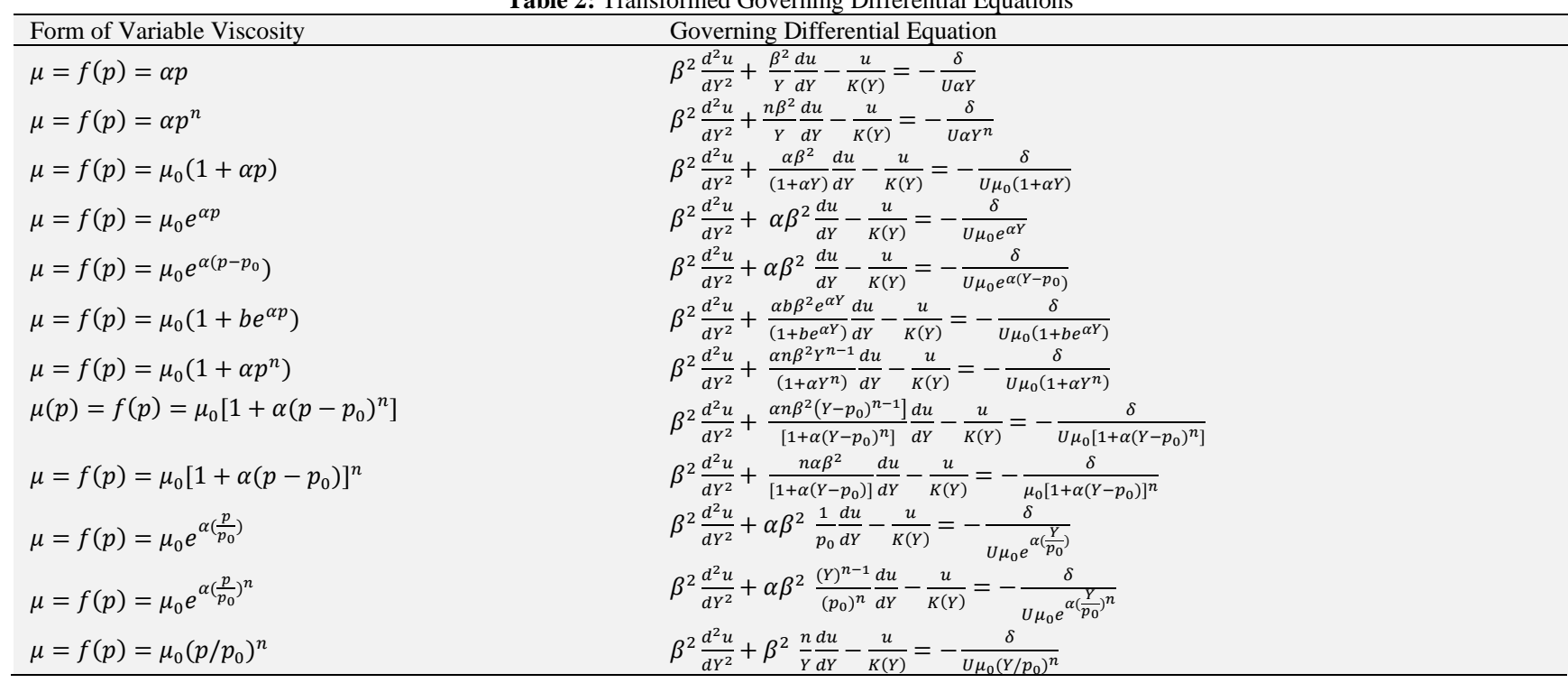

Solutions to the equations of Table 2 depend on the form of $K(Y)$. While we can always rely on a numerical solution, we will make the following assumption so that we can obtain closed form solutions to the equations of Table 2.

Hamdan and Kamel [22] introduced a permeability function for Brinkman's equation and showed that a quadratic velocity profile is tiled to a quadratic permeability. This translates into a proportionality between the variable permeability and velocity. We can then write $K(Y)=$ $k_{0} u$, where $k_{0}$ is a reference constant permeability.

Accordingly, equations of Table 2 are transformed into the following forms: 
Table 3: Final Form of Governing Differential Equations

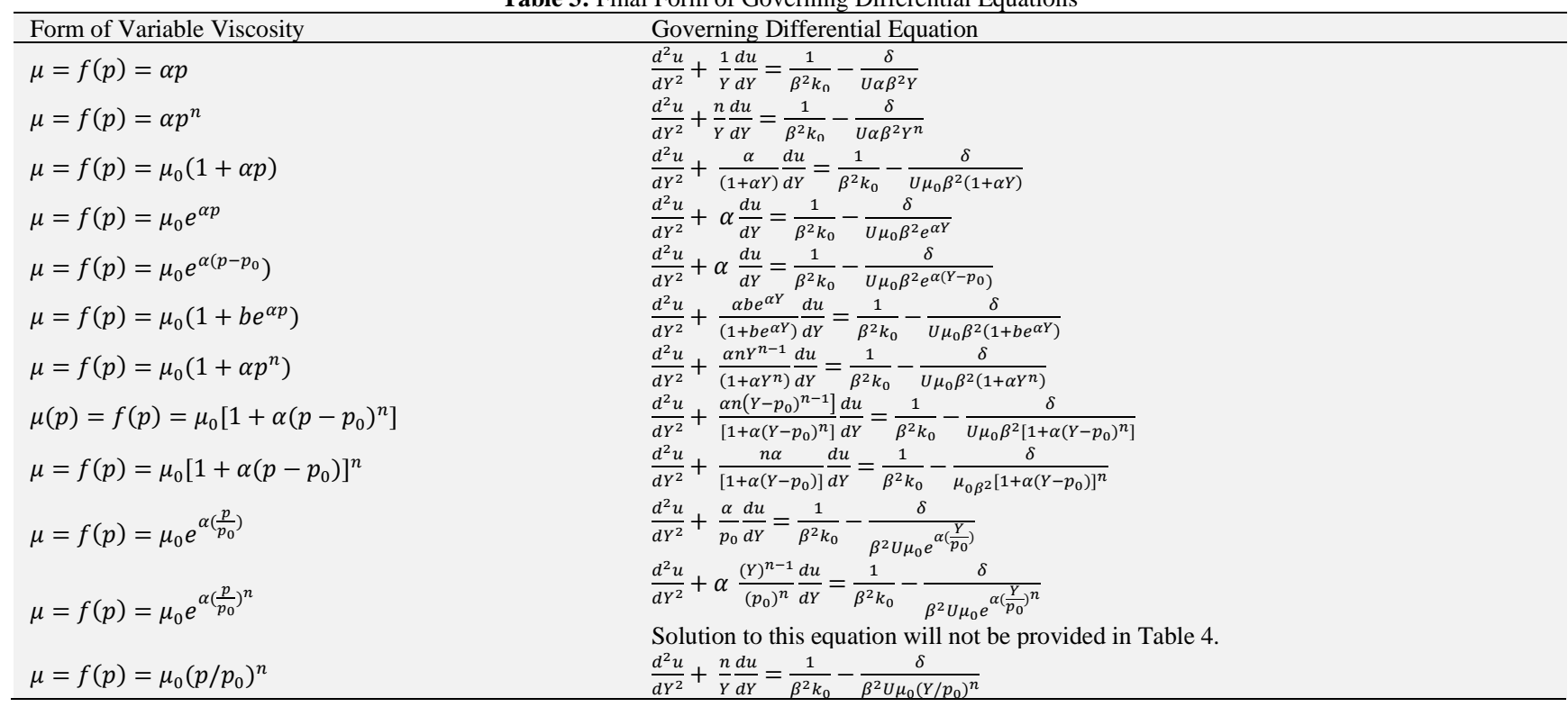

\subsection{Solutions to governing equations}

In order to solve the governing equations in Table 3, we use the method of reduction of order by letting $Z=\frac{d u}{d Y}$ and $\frac{d Z}{d Y}=\frac{d^{2} u}{d Y^{2}}$. Each of the differential equations is reduced to a first order, linear ODE of the form:

$\frac{d Z}{d Y}+f_{1}(Y) Z=f_{2}(Y)$

Equation (42) can be solved using integrating factor method. Solutions for the velocity distributions are shown in Table 4, below, where $c_{1}$ and $c_{2}$ are arbitrary constants to be determined using the no-slip conditions of (10) and (11).

Table 4: General Solutions to the Governing Differential Equations

\begin{tabular}{|c|c|}
\hline Form of Variable Viscosity & Governing Differential Equation \\
\hline$\mu=f(p)=\alpha p$ & $u=\frac{Y^{2}}{4 \beta^{2} k_{0}}-\frac{\delta Y}{U \alpha \beta^{2}}+c_{1} \ln Y+c_{2}$ \\
\hline$\mu=f(p)=\alpha p^{n}$ & $u=\frac{Y^{2}}{2(n+1) \beta^{2} k_{0}}-\frac{\delta Y^{2-n}}{U \alpha \beta^{2}(2-n)}+\frac{c_{1} Y^{1-n}}{1-n}+c_{2} ; n \neq 2$ \\
\hline$\mu=f(p)=\mu_{0}(1+\alpha p)$ & $u=\frac{Y^{2}}{4 \beta^{2} k_{0}}+\left[\frac{1}{2 \alpha \beta^{2} k_{0}}-\frac{\delta}{\alpha U \mu_{0} \beta^{2}}\right]\left[Y-\frac{1}{\alpha} \ln (1+\alpha Y)\right]+\frac{c_{1}}{\alpha} \ln (1+\alpha Y)+c_{2}$ \\
\hline$\mu=f(p)=\mu_{0} e^{\alpha p}$ & $u=\frac{Y}{\alpha \beta^{2} k_{0}}+\frac{\delta e^{-\alpha Y}}{U \mu_{0} \beta^{2}}\left[\frac{y}{\alpha}+\frac{1}{\alpha^{2}}\right]-\frac{c_{1} e^{-\alpha Y}}{\alpha}+c_{2}$ \\
\hline$\left.\mu=f(p)=\mu_{0} e^{\alpha\left(p-p_{0}\right.}\right)$ & $u=\frac{Y}{\alpha \beta^{2} k_{0}}+\frac{\delta e^{-\alpha Y}}{U \mu_{0} \beta^{2} e^{-\alpha p_{0}}}\left[\frac{y}{\alpha}+\frac{1}{\alpha^{2}}\right]-\frac{c_{1} e^{-\alpha Y}}{\alpha}+c_{2}$ \\
\hline$\mu=f(p)=\mu_{0}\left(1+b e^{\alpha p}\right)$ & 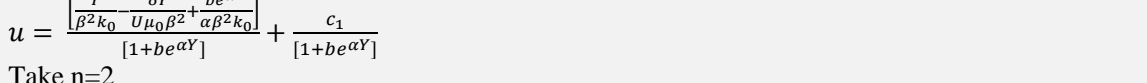 \\
\hline$\mu=f(p)=\mu_{0}\left(1+\alpha p^{n}\right)$ & $\begin{array}{l}u=\frac{c_{1}}{\sqrt{\alpha}} \tan ^{-1}(\sqrt{\alpha} Y)+\left[\frac{1}{2 \alpha \beta^{2} k_{0}}-\frac{\delta}{2 \alpha U \mu_{0} \beta^{2}}\right] \ln \left|1+\alpha Y^{2}\right|+\frac{1}{3 \beta^{2} k_{0}}\left[Y-\frac{1}{2 \alpha} \ln \left|1+\alpha Y^{2}\right|\right]+c_{2} \\
\text { Take } \mathrm{n}=2\end{array}$ \\
\hline $\begin{array}{l}\mu(p)=f(p)=\mu_{0}[1+\alpha(p- \\
\left.\left.p_{0}\right)^{n}\right]\end{array}$ & $\begin{array}{l}u=\frac{c_{1}}{\sqrt{\alpha}} \tan ^{-1}\left(\sqrt{\alpha}\left(Y-p_{0}\right)\right)+\left[\frac{1}{2 \alpha \beta^{2} k_{0}}-\frac{\delta}{2 \alpha U \mu_{0} \beta^{2}}\right] \ln \left|1+\alpha\left(Y-p_{0}\right)^{2}\right|+\frac{1}{3 \beta^{2} k_{0}}\left[\left(Y-p_{0}\right)-\frac{1}{2 \alpha} \ln \mid 1+\right. \\
\left.\alpha\left(Y-p_{0}\right)^{2} \mid\right]+c_{2}\end{array}$ \\
\hline$\mu=f(p)=\mu_{0}\left[1+\alpha\left(p-p_{0}\right)\right]^{n}$ & $u=\frac{Y\left(1-\alpha p_{0}\right)+\alpha \frac{Y^{2}}{2}}{\beta^{2} k_{0} \alpha(n+1)}-\frac{\delta}{\mu_{0 \beta^{2}}}\left\{\frac{\left[1+\alpha\left(Y-p_{0}\right)\right]^{2-n}}{\alpha^{2}(2-n)}+\left(p_{0}-\frac{1}{\alpha}\right) \frac{\left[1+\alpha\left(Y-p_{0}\right)\right]^{1-n}}{\alpha(1-n)}\right\}+c_{1} \frac{\left[1+\alpha\left(Y-p_{0}\right)\right]^{1-n}}{\alpha(1-n)} ; n \neq 1,2$ \\
\hline $\begin{array}{l}\mu=f(p)=\mu_{0} e^{\alpha\left(\frac{p}{p_{0}}\right)} \\
\mu=\mathrm{f}(\mathrm{p})=\mu_{0}\left(\mathrm{p} / \mathrm{p}_{0}\right)^{\mathrm{n}}\end{array}$ & $\begin{array}{l}u=\frac{Y}{\beta^{2} \mathrm{k}_{0} \frac{\alpha}{p_{0}}}-\frac{\delta}{\beta^{2} U \mu_{0}}\left[\frac{Y}{\frac{-\alpha}{p_{0}}}-\frac{\left(p_{0}\right)^{2}}{\alpha^{2}}\right] e^{\frac{-\alpha}{p_{0}} Y}+\frac{c_{1} e^{\frac{-\alpha}{p_{0}} Y}}{\frac{-\alpha}{p_{0}}}+c_{2} \\
u=\frac{Y^{2}}{2 \beta^{2} k_{0}(n+1)}-\frac{\delta\left(p_{0}\right)^{n}}{\beta^{2} U \mu_{0}(2-n)} Y^{2-n}+\frac{c_{1} Y^{1-n}}{1-n}+c_{2} ; n \neq 1,2\end{array}$ \\
\hline
\end{tabular}

Table 4 represents the entry profile to a porous channel the flow through which is governed by the generalized Brinkman's equation. For the case of $\mu=f(p)=\mu_{0} e^{\alpha\left(\frac{p}{p_{0}}\right)^{n}}$, the resulting differential equation needs to be solved numerically. For the eleven other popular expressions for viscosity in terms of pressure, we have provided a closed-form profile for the entry velocity.

\section{Conclusion}

In this work, we considered flow of a pressure-dependent viscosity fluid down an inclined porous plane with the objective of deriving entry velocity profiles to either a free-space channel or porous channel. This work is important in the study of two-dimensional flow through channels when the fluid is of a pressure-dependent viscosity. To achieve the said objective, we considered Navier-Stokes equations with 
pressure-dependent viscosity, and the generalized forms of Darcy, Forchheimer and Brinkman equations. We derived entry velocity expressions for a dozen cases of viscosity-pressure relations. From the above work, we can highlight the following findings.

a) In using the Barus viscosity-pressure relation, one needs an experimental Barus coefficient, $\beta_{\mathrm{B}}$.

b) Generalized Darcy's and Forchheimer's equations are not compatible with the presence of solid walls on which a no-slip velocity is imposed. In this work, we derived expressions for the slip velocities at the walls.

c) In using the generalized Brinkman's equation, a closed-form solution might not readily avail itself, depending on the form of viscosity and drag function in terms of pressure.

d) Generalized Brinkman's equation does not explicitly provide information on how to distinguish between one porous medium or another. In this work, we provided a methodology in which we tied the drag function to the medium variable permeability and obtained velocity expressions for a dozen viscosity-pressure relations.

The main emphasis in this work has been on flow through an inclined channel. We leave for future work to consider flow through a horizontal channel.

\section{References}

[1] G.G. Stokes, On the theories of the internal friction of fluids in motion, and of the equilibrium and motion of elastic solids, Trans. Camb. Philos. Soc. 8 (1845) 287-305.

[2] C.J. Barus, Note on dependence of viscosity on pressure and temperature, Proceedings of the American Academy 27 (1891) 13-19. https://doi.org/10.2307/20020462.

[3] C.J. Barus, Isothermals, isopiestics and isometrics relative to viscosity, American Journal of Science 45 (1893) 87-96. https://doi.org/10.2475/ajs.s345.266.87.

[4] S. Srinivasan, K.R. Rajagopal, A thermodynamic basis for the derivation of the Darcy, Forchheimer and Brinkman models for flows through porous media and their generalizations, International Journal of Non-Linear Mechanics 58 (2014)162-166. https://doi.org/10.1016/j.ijnonlinmec.2013.09.004.

[5] K.R. Rajagopal, G. Saccomandi, L. Vergori, Flow of fluids with pressure- and shear-dependent viscosity down an inclined plane, Journal of Fluid Mechanics 706 (2012) 173-189. https://doi.org/10.1017/jfm.2012.244.

[6] V.L. Savatorova, K.R. Rajagopal, Homogenization of a generalization of Brinkman's equation for the flow of a fluid with pressure dependent viscosity through a rigid porous solid, ZAMM 91\#8 (2011) 630-648. https://doi.org/10.1002/zamm.201000141.

[7] K. Kannan, K.R, Rajagopal, Flow through porous media due to high pressure gradients, Applied Mathematics and Computation 199 (2008) 748-759. https://doi.org/10.1016/j.amc.2007.10.038.

[8] P.W. Bridgman, The Physics of High Pressure, MacMillan, New York, 1931.

[9] A.Z. Szeri, Fluid Film Lubrication: Theory and Design, Cambridge University Press, 1998. https://doi.org/10.1017/CBO9780511626401.

[10] P.H. Vergne, Pressure-viscosity behavior of various fluids, High Press. Res. 8 (1991) 451-454. https://doi.org/10.1080/08957959108260704.

[11] F.J. Martinez-Boza, M.J. Martin-Alfonso, C. Callegos, M. Fernandez, High-pressure behavior of intermediate fuel oils, Energy Fuels 25 (2011) 5138 5144. https://doi.org/10.1021/ef200958v.

[12] K.B. Nakshatrala, K.R. Rajagopal, A numerical study of fluids with pressure-dependent viscosity flowing through a rigid porous medium, Int. J. Numer. Meth. Fluids 67 (2011) 342-368. https://doi.org/10.1002/fld.2358.

[13] L. Fusi, A. Farina, F. Rosso, Mathematical models for fluids with pressure-dependent viscosity flowing in porous media, Int. J. Engineering Science 87 (2015) 110-118. https://doi.org/10.1016/j.ijengsci.2014.11.007.

[14] K.D. Housiadas, G.C. Georgiou, R.I. Tanner, A note on the unbounded creeping flow past a sphere for Newtonian fluids with pressure-dependent viscosity, International Journal of Engineering Science 86 (2015) 1-9. https://doi.org/10.1016/j.ijengsci.2014.09.004.

[15] M.H. Hamdan, Single-phase flow through porous channels: A review. Flow models and channel entry conditions, Applied Mathematics and Computations 62 \#2\&3 (1994) 203-222. https://doi.org/10.1016/0096-3003(94)90083-3.

[16] S.C. Subramanian, K.R. Rajagopal, A note on the flow through porous solids at high pressures, Computers and Mathematics with Applications 53 (2007) 260-275. https://doi.org/10.1016/j.camwa.2006.02.023.

[17] S. Srinivasan, A. Bonito, K.R. Rajagopal, Flow of a fluid through a porous solid due to high pressure gradient, J. Porous Media 16 (2013) $193-203$. https://doi.org/10.1615/JPorMedia.v16.i3.20.

[18] J. Hron, J. Málek, K.R. Rajagopal, Simple flows of fluids with pressure-dependent viscosities, Proceedings of the Royal Society 457 (2001) $1603-$ 1622. https://doi.org/10.1098/rspa.2000.0723.

[19] J. Málek, K.R. Rajagopal, Mathematical properties of the solutions to the equations governing the flow of fluids with pressure and shear rate dependent viscosities, in: Handbook of Mathematical Fluid Dynamics, Elsevier, 2007.

[20] J. Chang, K.B. Nakashatrala, J.N. Reddy, Modification to Darcy-Forchheimer model due to pressure-dependent viscosity: consequences and numerical solutions, J. Porous Media 20\#3 (2017) 263-285. https://doi.org/10.1615/JPorMedia.v20.i3.60.

[21] I. Pažanin, M. C. Pereira, F. J. Suárez-Grau, Asymptotic approach to the generalized Brinkman's equation with pressure-dependent viscosity and drag coefficient, J. Applied Fluid Mechanics 9\#6 (2016) 3101-3107. https://doi.org/10.29252/jafm.09.06.25756.

[22] M.H. Hamdan, M.T. Kamel, Flow through Variable Permeability Porous Layers, Adv. Theor. Appl. Mech. 4\#3 (2011) 135-145. https://doi.org/10.1615/SpecialTopicsRevPorousMedia.v2.i2.80.

[23] S.M. Alzahrani, I. Gadoura, M.H. Hamdan, A Note on the flow of a fluid with pressure-dependent viscosity through a porous medium with variable permeability, J. Modern Technology and Engineering, 2\#1 (2017) 21-33. 